\title{
JOINT DISCUSSION 2 ABSTRACTS
}

Update to SOFA Report: Patrick Wallace, Rutherford Appleton Laboratory, Chilton, Didcot, Oxon OX11 0QX, UK

\begin{abstract}
Since the Colloquium 180 paper was presented, the draft SOFA Software Collection has been prepared for Board inspection, and procedures have been put in place for the Board's views to be registered. At the time of writing, votes from three Board members have been received, approving release of the routines; Catherine Hohenkerk (HM$\mathrm{NAO}$ ) is carrying out detailed testing of the draft collection, comparing the results with software used in the production of the almanacs.

The Capitaine et al. precession-nutation algorithm has been coded to SOFA standards and its output has been compared with results provided by the Observatoire de Paris. Supporting routines have been drafted which compute stellar angle from UT and decompose the precession- nutation matrix into classical forms. When the final IAU 2000A and 2000B precession-nutation models are announced, SOFA implementations will be added to the draft SOFA Software Collection.
\end{abstract}

Numerical simulation of reductions of microarcsecond relativistic effects in space astrometry: Kopeikin, S.M. (Missouri-Columbia University, USA); Yagudina, E.I, Vasilyev, M.V., Shuygina, N.V. (Institute of Applied Astronomy RAS, Russia); Yagudin, L.I. (Pulkovo, Russia)

\begin{abstract}
The accuracy of the astrometric observations using space optical interferometers is expected to be of the order of 1 microarcsecond. The processing of such precise measurements demands the using of a rigorous relativistic model (for example, Klioner, S.A., Kopejkin, S.M., Astron. J., 1992, 104, 897). Special software is developed for taking into account all effects of one microarcsecond level caused by post-Newtonian and post-post-Newtonian monopole perturbations. Observations received by space optical interferometers located on an artificial satellite are simulated. The satellite orbit parameters and telescope characteristics were chosen rather close to that of Hipparcos mission. The data analysis has been performed to improve coordinates of small amount of stars. The applied relativistic model did not show the valuable enlargement of the accuracy of the adjusted stellar parameters. Evidently this result arises from the strong correlations between parameters of stars and small value of addition relativistic effects. The implementation of the "real life" observational program has to improve the accuracy of the stars position determination (of the order of $10 \mu \mathrm{as}$ ). In the final solution it is assumed to use the Guide Star Catalogue as an input one.
\end{abstract}




\section{Relativistic Astrometry: Toward an Accurate Determination of}

Gamma: B. Bucciarelli et al., Astronomical Observatory of Torino., Italy.

Abstract. The experiments we performed on a scaled end-to-end simulation of the GAIA mission in a PPN relativistic framework make us confident that an error of $\sim 10^{-6}$ on the estimation of the $\gamma$ parameter is a conservative one, and even better results could be achieved by GAIA. We also developed a more traditional formulation of the GAIA observation equation, which considers relativistic effects at the post-Newtonian order. The advantage of using this formulation is that the effects of aberration, parallax, and gravitational light deflection are all explicit in the condition equation, and their statistical properties can be more easily investigated. By using this approach in combination with different GAIA simulations, we hope to determine an optimal reduction strategy for the most accurate determination of $\gamma$.

Constants and Models of Milliarcsecond Astrometry as Compared with Constants and Models of Arcsecond Astrometry: Y.B. Kolesnik, Institute of Astronomy of the Russian Academy of Sciences, 48 Piatnitskaya str., 109017 Moscow, Russia

Abstract. The approach of arcsecond astrometry based on unification of great number relatively low accurate individual measurements has been compared to this by microarcsecond astrometry established on quasiinstant precise measurements. Investigation of the Hipparcos system of proper motions has shown that its actual random accuracy is still much inferior to the model of proper motions provided by the ground-based arcsecond astrometry. There are also strong indications of the systematic regional errors of the Hipparcos system of positions and motions which lead to the drastic discrepancy of this system with all ground-based catalogues at different epochs. On the other hand, from analysis of worldwide optical observations of the Sun and inner planets in the 20th century the residual rotation of the Hipparcos system with respect to dynamical reference frame of about $0.30 \pm 0.03^{\prime \prime} / \mathrm{cy}$ has been discovered. The three principal characteristics of a reference system: actual random accuracy, systematic accuracy and the overall spin of the Hipparcos catalogue are not consistent with its claimed formal estimates. The adopted IAU 1998 model of the realisation of the ICRS in optical is recognized to be unsatisfactory, and should be revised before results of the next astrometric space mission become available. 
Progress in the Determination of Some Astronomical Constants from Radar Observations of Planets and Spacecraft: E.V. Pitjeva, Institute of Applied Astronomy of RAS, Russia

\begin{abstract}
Three main factors that influence the progress in the determination of astronomical constants - 1)necessary reductions, 2)dynamical models of planet motion, 3)observational data - are demonstrated. Reduction of measurements includes all needed corrections, so, including a reduction for Venus topography decreased the rms residuals of Venus observations by $23 \%$. The formal standard deviations of the solution elements of planets and AU are improved by $30-50 \%$ with using the DE405 or EPM2000 ephemerides, constructed in IAA (Russia) instead of DE200. It was shown that including measurements of the Viking and Pathfinder landers, being free from the uncertainties due to planetary topography, into the observational data reduces the uncertainties of adjusted parameters by 1-2 orders. The values of a broad set of astronomical constants, obtained in the fitting process of the DE405 and EPM2000 ephemerides to data totaling near 80,000 American and Russian radar observations of planets (1961-1997), the Viking and Pathfinder landers, are given.
\end{abstract}

A New Nutation Model of Non-rigid Earth with Ocean and Atmosphere: Cheng-li Huang, Wen-jing Jin and Xing-hao Liao, Shanghai Astronomical Observatory, NAO, CAS 80 Nandan Road, Shanghai 200030, P.R.China e-mail: clhuang@center.shao.ac.cn

\begin{abstract}
Using a completely numerical integration method, Huang et al. present a new nutation model of non-rigid Earth with ocean and atmosphere, in which incorporate all the second order terms in the boundary conditions and consider a readjustment of the flattening profile with respect to hydrostatic equilibrium, and include directly the oceanic load and current contribution for all nutation frequencies, as well as the atmospheric contribution in the integration.
\end{abstract}

How Present Precession-Nutation Models Agree with the Observations by Optical Astrometry and VLBI?: Jan Vondrák and Cyril Ron, Astronomical Institute, Academy of Sciences of the Czech Republic, Boční II, 14131 Prague 4, Czech Republic, e-mail vondrak@ig.cas.cz

Abstract. The series of celestial pole offsets obtained by the most recent optical astrometry solution OA00 on one side, and by Very LongBaseline Interferometry on the other, are compared with the standard 1980 IAU model of precession-nutation, and also with the recently proposed precession-nutation model MHB2000. The solution OA00 covers the interval 1899.7-1992.0 and is based on approximately 4.5 million individual observations made with 50 different instruments at 33 observatories. We concentrated only on longer periods - the values $\delta \psi, \delta \epsilon$ were first smoothed to suppress annual and shorter periodic variations, and 
then compared with the differences MHB2000-IAU1980. Although the optical astrometry solution still displays some systematic deviations, both curves generally follow approximately the same trend. Nevertheless, the trend of OA00 in $\delta \psi$ seems to be a little smaller than that of MHB2000. Systematic biases (larger for $\delta \psi$, smaller for $\delta \epsilon$ ) reflect the offset between the Celestial Intermediate Pole (CIP) from the Geocentric Celestial Reference System as defined by Resolution 7 of IAU Colloquium 180. Four different VLBI solutions are compared with the new model of precessionnutation: Goddard Space Flight Center (GSFC), Bundesamt für Kartografie und Geodäsie (BKG), Institute of Applied Astronomy (IAA) and U.S. Naval Observatory (USNO), all of them yielding very similar patterns. The biases in the sense observation minus (MHB2000BIAU1980) are more or less constant in time and nearly identical for all VLBI solutions. They are concentrated around -41.8 and -6.9 mas in $\delta \psi$ and $\delta \epsilon$ respectively. In order to detect the effect of free core nutation in the residuals, the apparently less noisy series of BKG are subject to spectral analysis. The spectrum is rather flat, and it is really very difficult to locate a peak corresponding to FCN.

The comparison of the newly proposed precession-nutation model MHB2000 with both optical astrometry (covering almost whole century) and VLBI (covering two decades) shows that the new model is no doubt much better than the present model IAU 1980. It agrees excellently with all available VLBI solutions, and its long-term is consistent, on a lower level of accuracy, also with optical astrometry. However, the effect of free core nutation is very weak and hardly detectable even from the most recent VLBI solutions, and our opinion is that it should not become a part of the new precession-nutation model.

\section{The Analysis of Pip and Blz Latitude Data and the Free Diurnal} Nutation: Goran Damljanovic, Astronomical Observatory, Volgina 7, 11160 Belgrade, Yugoslavia; Maria Silvina De Biasi Facultad de Ciencias Astronomicas y Geofisicas (UNLP) Paseo del Bosque s/n, 1900 La Plata, Argentina Consejo Nacional de Investigaciones Cientificas y Tecnicas, Argentina

Abstract. A recent advantages in the spatial and the temporal resolution of the Earth orientation parameters (EOP), regularly published by the International Earth Rotation Service (IERS), and the possibility to separate high-frequency polar motion and nutation, between each other, give us the chance to calculate (and to compare with the results of new technics) the values of the period (we calculated 0.61 years from PIP data and 0.58 years from BLZ ones), the amplitude (0.012 arcseconds from PIP, 0.014 arcseconds from BLZ) and the phase (300 degrees from PIP, 320 degrees from BLZ; epoch: 1900.0 years) of the free diurnal nutation by using 13 years of the Punta Indio (PIP data with longitude $=57$ degrees 17 minutes 10 arcseconds $\mathrm{W}$ and latitude $=-35$ degrees 20 minutes 40 arcseconds, in the HIPPARCOS reference frame) and 37 years of the Belgrade (BLZ data with longitude $=20$ degrees 30 minutes 48 arcseconds $\mathrm{E}$ and latitude $=+44$ degrees 48 minutes 10 arcseconds, in the 
FK5 reference frame) homogeneized latitude series after new reduction of both data. Both reductions are in line with MERIT Standards, with the standard IAU 1976 precession and IAU 1980 nutation. Direct Fourier transforms method of spectral analysis was applied to these optical astrometry data (PIP and BLZ), and the results presented here (compared with each other and with the other ones) can be useful (because of long history and new reduction of the past PIP and BLZ data) for some remarks of IAU 1980 nutation model and polar motion.

Numerical Theory of Non-rigid Earth: T. Shirai et al., University of Tokyo, Japan
Abstract. We determined the dynamical ellipticity of the Earth through the numerical theory of precession and nutation. We did full integration of Euler equation and solved procession and nutation simultaneously. We used JPL/NASA ephemeris DE405 for the planetary ephemeris and as- tronomical constants. We also used contemporary VLBI data complied by USNO for the period 1979-2000. In conclusion we estimated dynam- ical ellipticity $H$ as $0.0032737914(4)$, which is a little bigger than those estimated analytically.

\title{
Proper Motions of 16,731 Stars Deduced from 1.5 Century Observa- tions, Decl > +75 Degrees: V.Tel'nyuk-Adamchuk, O.Gregul, and O.Molotaj, Astronomical Observatory of National Taras Shevchenko University of Kyiv
}

\begin{abstract}
Fourteen meridian and photographic catalogues, epoch span from 1855 to 1990 s were used to compile a catalogue of proper motions of 16731 stars, Decl $>75$ degree declination (authors: V.Tel'nyukAdamchuk, O.Gregul, and O.Molotaj; Astronomical Observatory of National Taras Shevchenko University of Kyiv). The source catalogues were converted to the Tycho2 (T2) systemby applying systematic differences. To determine them, in a 1 degree-radius circle centred at each source catalogue (SC) star, individual T2-SC differences were weighted in this circle and then used as a systematic correction for SC star to transform the star coordinates to T2 frame. Magnitude difference were also obtained and removed from SCs. After data final testing and cleaning, the proper motions and their uncertainties were computed using the SC weights. A comparison of deduced motions with those of $\mathrm{T} 2$ shows no apparent systematic biases between $\mathrm{T} 2$ and Kyiv proper motions. The r.m.s. uncertainties are equal to $1.3 \mathrm{mas} / \mathrm{yr}$. The results can be applied to maintain Hipparcos system in North polar region. Since the quality of Pulkovo photographic plates taken within the AGK2 program was estimated as being rather good, the Kyiv team started re-measuring and re-reducing the Pulkovo plates and plans to extract from the plates the positions of about $100 \mathrm{~K}$ stars, Decl $>+67.5$ degree, with an acceptable accuracy.
\end{abstract}




\title{
Systematic and Local Variations of Ground-Based Proper-Motion Sys-
} tems to Hipparcos: Zi Zhu and Tinggao Yang, Shaanxi Astronomical Observatory, P.O. Box 18, Lintong, China; zhuzi@ms.sxso.ac.cn

\begin{abstract}
On the basis of the Hipparcos data, whose system is believed to be quasi-inertial to within \pm 0.25 mas $\mathrm{yr}^{-1}$ and to represent an internally consistent reference frame, we have performed analyses on the FK5 proper-motion system via three large astrometric catalogues, the PPM, ACRS and the IRS catalogues, which were constructed on the FK5 system. The regional differences of proper motions between the PPM and Hipparcos, between the ACRS and Hipparcos, and between the IRS and Hipparcos, show a remarkably large residual velocity around the axis from the south celestial pole to the north celestial pole. The regional differences on the southern hemisphere are larger than those on the northern part. Considering the magnitude- and color-dependent pattern of the propermotion difference, we have found that the PPM and ACRS catalogues suffer a quite similar fashion in their color and magnitude equations with respect to the Hipparcos proper-motion system. Comparing the Hipparcos proper motions with those of the SPM 2.0 Catalog, which provides absolute proper motions of objects measured directly relative to external galaxies, we found that the regional differences of the SPM 2.0 proper motions exhibit neither strong systematics nor large regional errors. The typical regional error for the SPM 2.0 proper motions is favorably as small as $=\mathrm{B} 10.8$ mas yr-1. The global rotation related to the Hipparcos frame is slower than 0.25 mas yr-1 except the component along the $y$-axis which is evaluated as large as a factor 2 than this value for the uncertainty of the Hipparcos inertiality. Inspecting the magnitude- and color-equations between the two proper-motion systems, we found that the proper-motion differences between the SPM 2.0 and Hipparcos is slightly correlated with the color indices of stars, but no clear magnitude-equation are found.
\end{abstract}

Non-Stability of the Chandler Wobble Excitation: N.S. Sidorenkov, Hydrometcentre of Russia

Abstract. A new concept on the interactions in the Earth-Ocean-Atmosphere system was formulated. A Chandler wobble excitation model was developed based on the non-linear oscillations in the Earth-OceanAtmosphere system. The presentation showed that the decade time fluctuations in the intensity of the ENSO lead to instabilities in the Chandler wobble excitation process, and thus to the decade variations in the Chandler wobble characteristics (amplitude, phase, damping decrement, etc.). 
Determination of Optical Positions for Extragalactic Radio Sources under the Collaboration Between SHAO and NAO: Z. H. Tang, W. J. Jin and S. H. Wang, Shanghai Astronomical Observatory, Chinese Academy of Sciences, Shanghai 200030, China; G. Pinigin, A. Shulga, N. Maigurova, Yu. Protsyuk Nikolaev Astronomical Observatory, Nikolaev, 327030, Ukraine

\begin{abstract}
The optical positions of 9 compact extragalactic radio sources by using $1.56 \mathrm{~m}, 1 \mathrm{~m}$ and $60 / 90 \mathrm{~cm}$ telescope with CCD in China and the Axial Meridian Circle at Nikolaev Astronomical Observatory have been determined since Jan. 1996 under a collaboration project between two observatories. The instrumentation, observation and reduction are briefly described and the preliminary results are presented. The comparison between the optical and radio positions for 9 sources are also given. More optical positions of radio sources are being processed.
\end{abstract}

\title{
Linking Radio-Optical Celestial Reference Frames with Using of CCD
} Astrographs and Nikolaev Automatic Axial Meridian Circle: G.Pinigin, A.Shulga, Nikolaev, Astronomical Observatory (Ukraine)

\begin{abstract}
The previous results of collaborated programme between three observatories from China, Russia and Ukraine for refinement of the optical/radio reference frames linking were discussed. The optical position of several extragalactic radio sources (ERS) were determined by using of CCD telescopes with accuracy on 20-70 mas level. As reference catalogue was used one consisted of 14000 stars selected from the USNO-A2.0 in the fields around 200 ERS. This catalogue was received from observations with Nikolaev Axial meridian circle for declination zone from equator to +70 in the 12-14.5 magnitude range during $1996-1998$. It was estimated an expected accuracy of the optical/radio linking for case of using about 200 ERS for northern declination zone on the level of 5 mas. For this purpose it needs to provide of ERS optical position accuracy not worse than 20 mas.
\end{abstract}

Comment on the New Definition of the Celestial Ephemeris Pole: Ya. Yatskiv, Main Astronomical Observatory of National Academy of Science of Ukraine

Abstract. Ya. Yatskiv has discussed the Resolutions 7 and 8 of the IAU Coll. 180 concerned with definitions of the CIP and the CEO. Noting the various approaches for these definition he suggested very simple type of decomposition between nutation and polar motion, namely the nutation is long-periodical predicted forced motion of the CEP with respect to the CRS, and all other components of nutation (diurnal and sub-diurnal as well as so-called CPO) should be considered as motion of the CEP with respect to the TRS.

He also suggested to combine the two resolutions mentioned above into single one defining the axis of the Intermediate Reference System. 